\title{
Proof of concept in cardiovascular risk: the paradoxical findings in blood pressure and lipid abnormalities
}

This article was published in the following Dove Press journal:

Vascular Health and Risk Management

II July 2012

Number of times this article has been viewed

\author{
Flavio Danni Fuchs \\ Sandra Costa Fuchs \\ Leila Beltrami Moreira \\ Miguel Gus \\ Division of Cardiology and \\ Postgraduate Studies Program \\ in Cardiology, Hospital de Clinicas \\ de Porto Alegre, Universidade Federal \\ do Rio Grande do Sul, Porto Alegre, \\ Rio Grande do Sul, Brazil
}

\begin{abstract}
High blood pressure and lipoprotein abnormalities were identified by many cohort studies as the major risk factors for cardiovascular disease. Laboratory experiments apparently confirmed their role in the causation of atherosclerosis, but a proof of concept requires the corroboration by clinical trials in human beings. The size of benefit in clinical trials regarding the control of high blood pressure was within the estimations of risk provided by cohort studies. For a reduction of $10 \mathrm{mmHg}$ in systolic blood pressure or $5 \mathrm{mmHg}$ in diastolic blood pressure, the relative risk reduction of coronary heart disease was $22 \%(95 \%$ confidence interval $27 \%-17 \%)$ in a meta-analysis of clinical trials, close to the estimation of reduction of $25 \%(95 \%$ confidence interval $23 \%-27 \%$ ) provided by a meta-analysis of cohort studies. The corresponding values for stroke were $41 \%$ (95\% confidence interval 33\%-48\%) in clinical trials compared to a cohort risk prediction of 36\% (95\% confidence interval 34\%-38\%). This efficacy was shared by all blood pressure-lowering drugs. The same figure has not paradoxically happened with drugs that act over abnormalities of cholesterol and lipoproteins. Only statins, which have other beneficial actions as well, have consistently lowered the incidence of cardiovascular diseases, an efficacy that was not reproduced by older and newer quite potent lipid drugs. The adverse effects of these drugs may nullify their beneficial effects over lipoproteins and abnormalities of lipoproteins may only be surrogate markers of the underlying real risks.
\end{abstract}

Keywords: proof of concept, hypertension, lipoproteins, clinical trials

\section{Introduction}

Classic cohort studies identified high blood pressure and blood lipids abnormalities as major risk factors for cardiovascular disease. Nonetheless, their role in the causation of cardiovascular disease - the proof of concept - requires corroboration by clinical trials. Findings from clinical trials regarding the control of high blood pressure have been consistent with the estimations of risk provided by cohort studies, but the same has not paradoxically happened with drugs that act on abnormalities of cholesterol and lipoproteins. In this descriptive review, the key studies of risk and benefit of treatment that support this view are presented.

\section{Risks of blood pressure}

The meta-analysis of 61 cohort studies by the Prospective Studies Collaboration group, with more than 1 million individuals at risk and with more than 53,000 fatal strokes and coronary events during a follow-up of more than 15 years, identified that the risks start at blood pressure values as low as 115/75 $\mathrm{mmHg}$, doubling at each $20 \mathrm{mmHg}$ of systolic blood pressure or $10 \mathrm{mmHg}$ of diastolic blood pressure (Figure 1). The play
Correspondence: Flavio Danni Fuch Clínicas de Porto Alegre, Ramiro Barcelos 2350, Porto Alegre 90035-903, Rio Grande do Sul, Brazil

Tel +55 5I 33598420

Fax +55 51 33598420

Email ffuchs@hcpa.ufrgs.br 

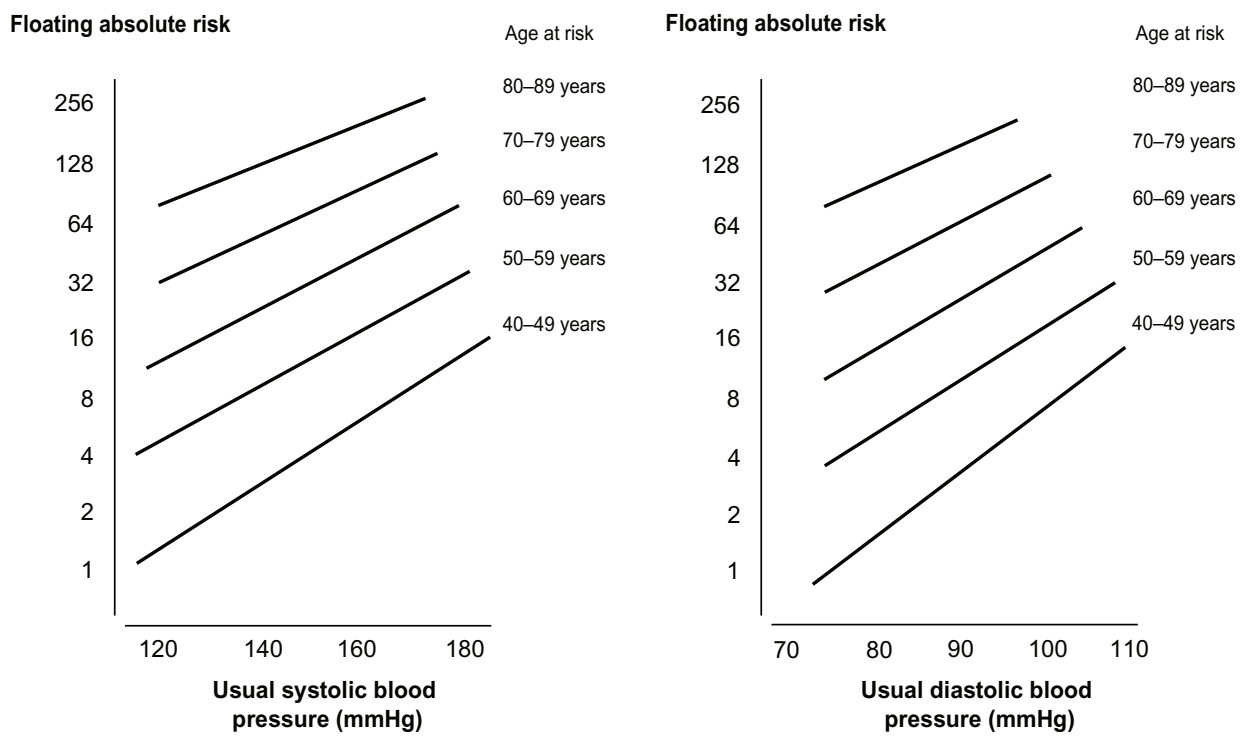

Figure I Absolute risk for coronary heart disease by age and usual systolic and diastolic blood pressure.

Reproduced with permission from The Lancet, Lewington S, Clarke R, Qizilbash N, Peto R, Collins R. Age-specific relevance of usual blood pressure to vascular mortality: a meta-analysis of individual data for one million adults in 61 prospective studies. Lancet. 2002;360(9349):1903-1913.' Copyright 2002, with permission from Elsevier.

of chance was probably close to null in face of the large number of individuals included in the meta-analysis, and selection and measurement bias of the individual studies were essentially diluted as well.

\section{Risks of lipid abnormalities}

Using a methodology similar to the blood pressure metaanalysis, the investigators of the Prospective Studies Collaboration analyzed the association of cholesterol and lipoproteins with deaths by coronary heart disease (CHD) and stroke, based on 61 cohort studies, with almost 900,000 adults and with more than 55,000 vascular deaths in the follow-up. ${ }^{2}$ The association between cholesterol levels with the incidence of CHD deaths was exponential, continuous, and observed at all age groups, reproducing the findings of blood pressure (Figure 2). Low-density lipoprotein cholesterol (LDL-C) was positively associated, and high-density lipoprotein cholesterol (HDL-C) was inversely associated, with the incidence of coronary events. Differently from blood pressure, which was a risk for CHD and stroke, cholesterol levels (and fractions) were not associated with the incidence of stroke, particularly among individuals with high blood pressure and older ages.

\section{Requirements for a proof of concept} Despite the robust evidence, observational studies are not sufficient to provide a proof of concept since it is not possible to control for many potential biases on the relationship between exposures and events. Some confounding factors were controlled by stratification and statistical modeling, but others were difficult to measure or were not addressed in all studies, such as genetic traits, psychosocial characteristics, and other unknown confounders. Some characteristics may be only surrogates of risks and not the real risks. Only experiments can provide the proof of concept. Since human beings cannot be experimentally exposed to potential risks

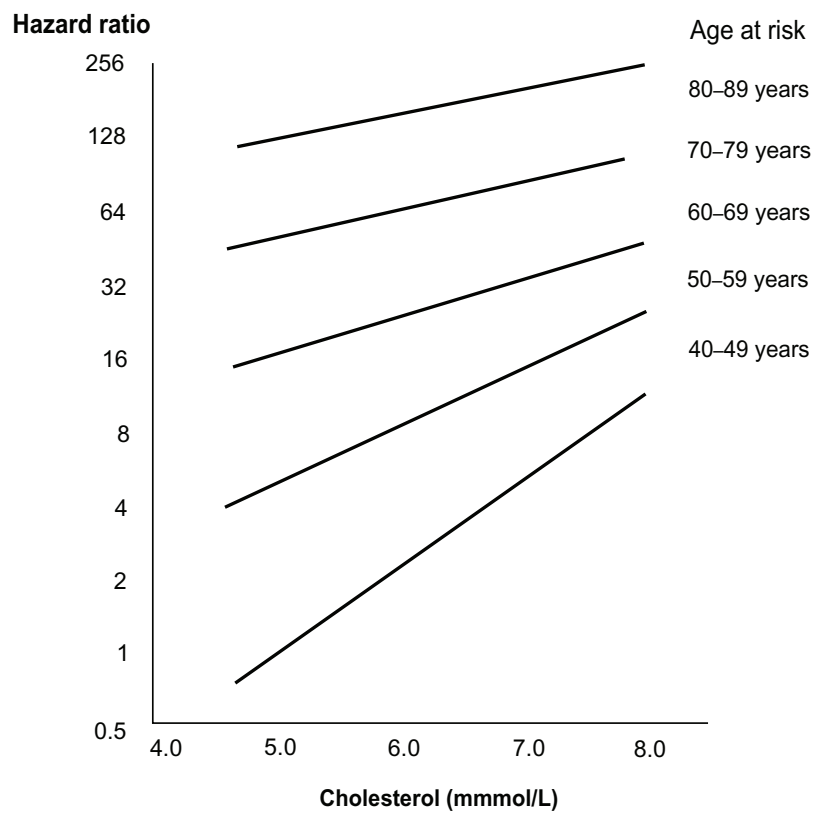

Figure 2 Absolute risk for coronary heart disease by age and serum cholesterol levels.

Reproduced with permission from The Lancet, Lewington S, Whitlock G, Clarke R, et al; Prospective Studies Collaboration. Blood cholesterol and vascular mortality by age, sex, and blood pressure: a meta-analysis of individual data from 61 prospective studies with 55,000 vascular deaths. Lancet. 2007;370(9602):1829-1839.2 Copyright 2007, with permission from Elsevier. 
or causes for diseases, the proof of concept can be given only by indirect experiments, which try to demonstrate if diseases are prevented by removal or antagonism of potential risks.

Most, but not all, risks and benefits identified in observational studies have been corroborated by clinical trials. The prevention of cardiovascular disease by hormone replacement therapy after menopause, demonstrated in cohort studies and not confirmed by clinical trials, is a noticeable example. 3,4

\section{Proof of concept for hypertension}

The causal role of high blood pressure for cardiovascular disease was fully confirmed by clinical trials. Compiling more than a hundred clinical trials, Law and associates demonstrated the robust association between lowering of blood pressure and the prevention of CHD and stroke. ${ }^{5}$ The summary estimate and confidence intervals (CI) for the prevention of CHD (relative risk $0.78,95 \%$ CI: 0.73-0.83) were almost those predicted by the cohort meta-analysis (relative risk 0.75, 95\% CI: 0.73-0.77) (Figure 3A). ${ }^{1}$ For

\section{A}

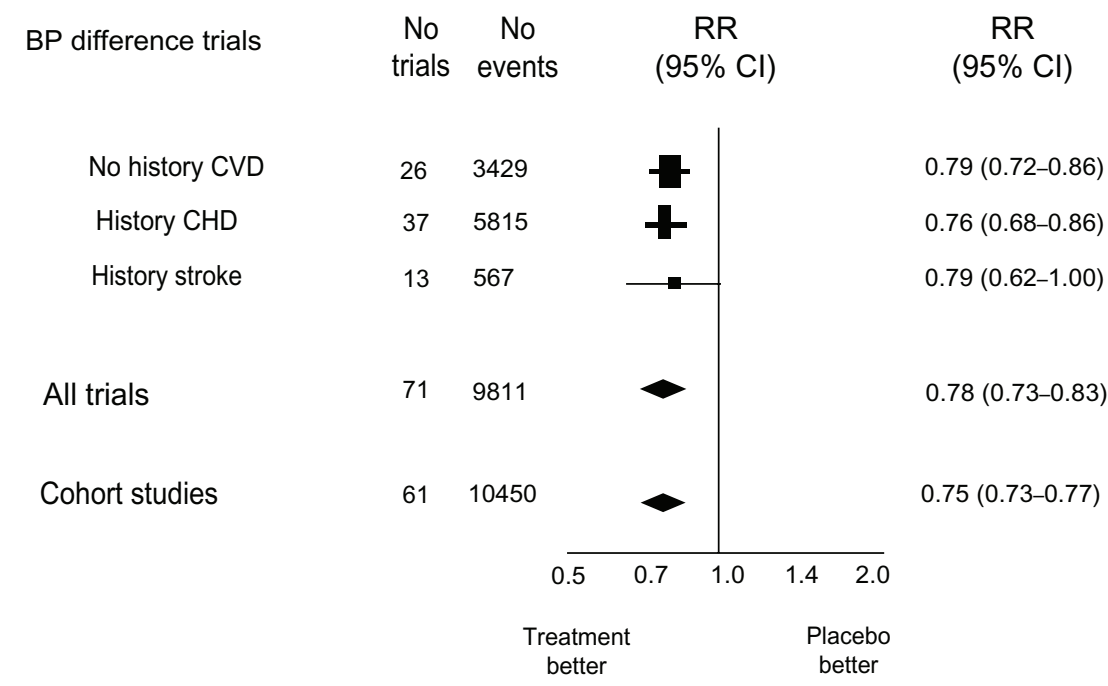

B

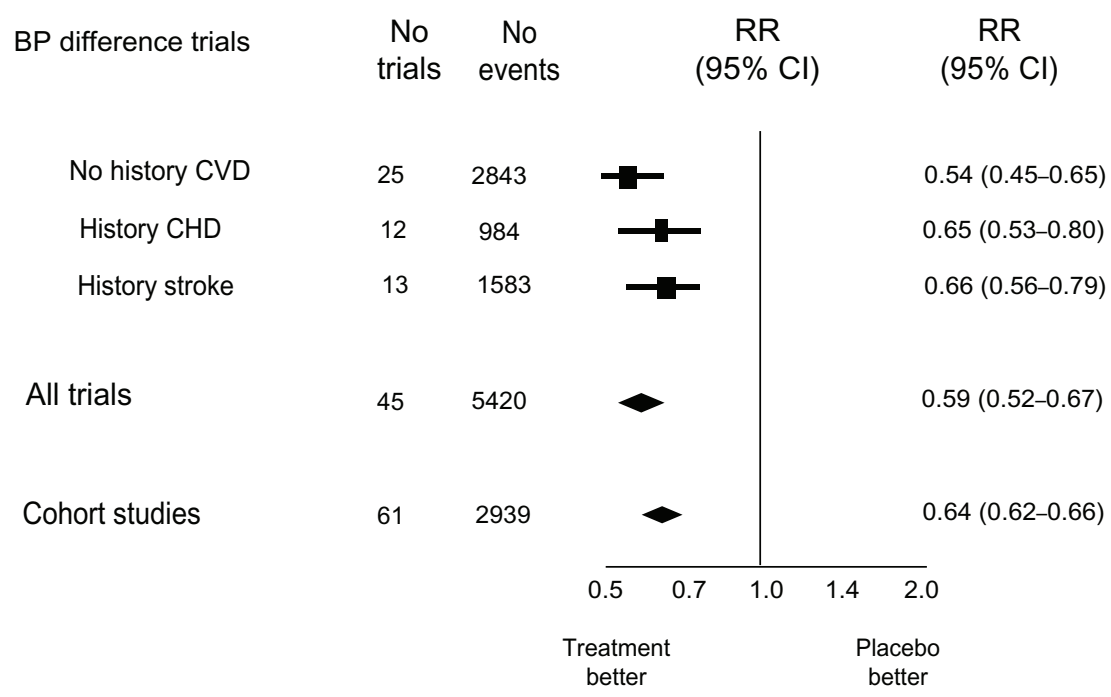

Figure 3 Relative risk for $(\mathbf{A})$ coronary heart disease and (B) stroke by standardized difference ( $10 \mathrm{mmHg}$ of systolic blood pressure or $5 \mathrm{mmHg}$ of diastolic blood pressure) between clinical trial arms in patients with and without previous cardiovascular disease, and the size effect prediction from cohort studies.

Reproduced with permission from BMJ Publishing Group Limited. Law MR, Morris JK, Wald NJ. Use of blood pressure lowering drugs in the prevention of cardiovascular disease: meta-analysis of 147 randomised trials in the context of expectations from prospective epidemiological studies. BMJ. 2009;338:bI665. ${ }^{5}$ Copyright 2009.

Abbreviations: BP, blood pressure; CHD, coronary heart disease; $\mathrm{Cl}$, confidence interval; $\mathrm{CVD}$, cardiovascular disease; RR, relative risk. 
stroke, the evidence was similar but of higher magnitude in face of the higher risk of blood pressure for cerebrovascular events (relative risk $0.59,95 \% \mathrm{CI}: 0.52-0.67$ ) in the meta-analysis of the clinical trials in comparison with that predicted by the cohort meta-analysis (relative risk 0.64, 95\% CI: 0.62-0.66) (Figure 3B). ${ }^{1}$ All drugs tested in the trials lowered the incidence of cardiovascular events with slight variation between them, demonstrating that their blood pressure-lowering properties, and not pleiotropic effects, were responsible for cardiovascular protection.

\section{Lack of proof of concept for lipid abnormalities}

A proof of evidence similar to that provided for the risks of high blood pressure was still not yielded for abnormalities of cholesterol and lipoproteins. Many drugs that are effective in improving the lipid profile did not lead to the expected reduction in the incidence of cardiovascular disease. The attempt to demonstrate the efficacy of the first lipid-lowering agents to prevent cardiovascular events in pioneering clinical trials was frustrating. Finnish executives with high risk of suffering a cardiovascular event and submitted to a multifactorial intervention, which included the use of probucol, clofibrate, or niacin, had higher incidence of CHD in comparison with participants randomized to usual care. The harmful effect of intervention persisted for 10 years after concluding the experimental phase. ${ }^{6}$ In the Lipid Research Clinic trial, the prevention of CHD in patients treated with cholestyramine reached statistical significance only with the use of a controversial one-sided alpha error test. ${ }^{7}$ Niacin was the only agent tested in the Coronary Drug Project that showed a trend for efficacy. ${ }^{8}$ A meta-analysis of 22 trials showed that old lipid-lowering agents failed to prevent cardiovascular events. ${ }^{9}$ Moreover, older lipid drugs were associated with higher risk for mortality by accidents and violence, ${ }^{10}$ leading to the discredit of the beneficial effects of lipid-lowering interventions at that point in time. ${ }^{11}$

The efficacy of statins to prevent cardiovascular events seemed to have settled the controversy about the effectiveness of lipid-lowering drugs to prevent cardiovascular disease, since they reduced the incidence of CHD mortality by $30 \%$ and total mortality by $12 \% .{ }^{12} \mathrm{~A}$ similar protection against stroke was somewhat unexpected, since higher cholesterol is not an evident risk factor for cerebrovascular events. ${ }^{13}$ A recent meta-analysis done by investigators of the authors' institute employed an innovative approach - mixed treatment comparisons - to compare the efficacy of different doses of statins. ${ }^{14}$ Based on the results of 47 clinical trials, totalizing 175,232 patients, they demonstrated that intermediate and higher doses were more efficacious in the prevention of cardiovascular events than lower doses. On the other hand, larger doses were not superior to prevent against cardiovascular mortality. The efficacy of statins in the primary prevention of cardiovascular events in patients with low risk has been questioned because of the inclusion of people with cardiovascular disease in some primary prevention trials. ${ }^{15}$ Many putative beneficial effects of statins have not been confirmed. ${ }^{16,17}$

The inefficacy of newer lipid-lowering agents introduced after statins renewed the uncertainty about the cardiovascular risks of higher cholesterol and other lipid abnormalities. Despite their intense effect over lipoproteins, newer drugs have failed to prevent major and surrogate cardiovascular outcomes. Torcetrapib increased HDL-C by $60 \%$ and reduced LDL-C by $20 \%$ (in addition to the effect obtained with a statin), but increased total mortality by $58 \%{ }^{18}$ Ezetimibe reduced LDL-C by approximately $16 \%$ in addition to the effect of statins, but failed to reduce the carotid intima-media thickness in patients with familial hypercholesterolemia. ${ }^{19}$ Compared to niacin, ezetimibe did not reduce carotid intima-media thickness and was associated with higher incidence of cardiovascular outcomes. ${ }^{20}$ Fenofibrate, another agent with moderate effect over cholesterol and intense effect over triglycerides, failed to prevent major cardiovascular events in patients with type 2 diabetes. ${ }^{21}$ The dissociation between the effect of drugs over cholesterol and fractions and over clinical outcomes is shown in Table 1.

Explanations for the beneficial effects of statins and maybe niacin and for the null or harmful effects of other agents have been presented. The size and precocious benefits of statins suggested they could act by other mechanisms, which were also aggregated under the denomination of pleiotropic effects. ${ }^{22}$ A blood pressure-lowering effect of statins has been suggested as well. ${ }^{23}$ In clinical trials, statins decreased cardiovascular outcomes to the same extent in hypertensive and nonhypertensive patients, ${ }^{24}$ but the benefit of blood pressure-lowering effect has been identified in patients without hypertension as well. ${ }^{25}$

The deleterious effects of torcetrapib were attributed to the increase of blood pressure by $4 / 2 \mathrm{mmHg}$ during the course of the ILLUMINATE (Investigation of Lipid Level Management to Understand its Impact in Atherosclerotic Events) trial. ${ }^{18}$ Anacetrapib, a newer cholesteryl ester transfer protein inhibitor free of the blood pressure-increasing effect, was tested in a small safety trial. ${ }^{26}$ LDL-C was lowered by $39.8 \%$, while HDL-C increased by $138.1 \%$ beyond that seen in the placebo group. Blood pressure and adverse 
Table I Effect of agents that interfere on lipid metabolism and consequences on surrogate and hard outcomes. Based on data from previous studies $8,12,18-21$

\begin{tabular}{|c|c|c|c|c|}
\hline & $\begin{array}{l}\text { HDL- } \\
\text { cholesterol }\end{array}$ & $\begin{array}{l}\text { LDL- } \\
\text { cholesterol }\end{array}$ & $\begin{array}{l}\text { Carotid intima- } \\
\text { media thickness }\end{array}$ & $\begin{array}{l}\text { Cariovascular } \\
\text { events }\end{array}$ \\
\hline Statins & $\rightarrow$ & $\downarrow \downarrow \downarrow$ & Reduce & Prevent \\
\hline Ezetimibe & $\uparrow$ & $\downarrow \downarrow$ & Neutral & Unknown \\
\hline Torcetrapib & $\uparrow \uparrow \uparrow \uparrow$ & $\downarrow \downarrow$ & Neutral & Increase \\
\hline Niacin & $\uparrow \uparrow$ & $\downarrow$ & Reduce & Prevent \\
\hline Fenofibrate ${ }^{a}$ & $\uparrow \uparrow$ & $\downarrow \downarrow$ & NA & Null \\
\hline
\end{tabular}

Note: apromotes substantial lowering of triglycerides.

Abbreviations: HDL, high-density lipoprotein; LDL, low-density lipoprotein; NA, not available.

events did not rise in participants treated with anacetrapib and there was no beneficial trend for prevention of clinical events, despite the huge effect over lipoproteins. The beneficial effects of niacin could also be secondary to its blood pressure-lowering properties, identified in a post hoc analysis of a lipid trial. ${ }^{27}$

On the other hand, the absence of benefit of most lipidlowering drugs may be secondary to the complex relationship between blood lipid abnormalities and vascular risk. In comparing the contribution of hypertension to cardiovascular events, the effect of circulation lipid on the cardiovascular system is more complicated and it is the nature of the complexity that determines the difficulty of drug development. Other factors may also share the deleterious pathway that lipid causes cardiovascular damage. The idea that the correction of such abnormalities would lead to the prevention of cardiovascular disease may be quite simplistic, and innovative approaches to control lipid abnormalities may be required.

\section{Conclusion and perspective}

The evidence of risks of high blood pressure and the consistent reduction of such risks by clinical trials of blood pressure-lowering agents are robust proofs of the concept that high blood pressure is a major cardiovascular risk. The lack of equivalent evidence with drugs that lower cholesterol (and LDL-C) or increase HDL-C leads to two interpretations: (1) adverse events of these drugs nullify their beneficial effects over lipoproteins, or (2) abnormalities of lipoproteins are only surrogate markers of the underlying real risks. In any case, drugs cannot be indicated to prevent cardiovascular events based exclusively on their effects over lipoproteins.

\section{Acknowledgments}

This study was supported, in part, by the National Institute of Science and Technology for Health Technology Assessment (IATS) - CNPq/Brazil.

\section{Disclosure}

The authors report no conflicts of interest in this work.

\section{References}

1. Lewington S, Clarke R, Qizilbash N, Peto R, Collins R. Age-specific relevance of usual blood pressure to vascular mortality: a meta-analysis of individual data for one million adults in 61 prospective studies. Lancet. 2002;360(9349):1903-1913.

2. Lewington S, Whitlock G, Clarke R, et al; Prospective Studies Collaboration. Blood cholesterol and vascular mortality by age, sex, and blood pressure: a meta-analysis of individual data from 61 prospective studies with 55,000 vascular deaths. Lancet. 2007;370(9602):1829-1839.

3. Grady D, Herrington D, Bittner V, et al. Cardiovascular disease outcomes during 6.8 years of hormone therapy: Heart and Estrogen/ progestin Replacement study follow-up (HERS II). JAMA. 2002;288(1): 49-57.

4. Rossouw JE, Anderson GL, Prentice RL, et al. Risks and benefits of estrogen plus progestin in healthy postmenopausal women: principal results from the Women's Health Initiative randomized controlled trial. JAMA. 2002;288(3):321-333.

5. Law MR, Morris JK, Wald NJ. Use of blood pressure lowering drugs in the prevention of cardiovascular disease: meta-analysis of 147 randomised trials in the context of expectations from prospective epidemiological studies. BMJ. 2009;338:b1665.

6. Strandberg TE, Salomaa VV, Naukkarinen VA, Vanhanen HT, Sarna SJ, Miettinen TA. Long-term mortality after 5-year multifactorial primary prevention of cardiovascular disease in middle-aged men. JAMA. 1991; 266(9):1225-1229.

7. Lipid Research Clinics Program. The Lipid Research Clinics Coronary Prevention Trial results. I. Reduction in incidence of coronary heart disease. JAMA. 1984;251(3):351-364.

8. Coronary Drug Project. Clofibrate and niacin in coronary heart disease. JAMA. 1975;231(4):360-381.

9. Ravnskov U. Cholesterol lowering trials in coronary heart disease: frequency of citation and outcome. BMJ. 1992;305(6844):15-19.

10. Muldoon MF, Manuck SB, Matthews KA. Lowering cholesterol concentration and mortality: a quantitative rewiew of primary prevention trials. BMJ. 1990;301(6747):309-314.

11. Oliver MF. Prevention of coronary heart disease - propaganda, promises, problems, and prospects. Circulation. 1986;73(1):1-9.

12. Brugts JJ, Yetgin T, Hoeks SE, et al. The benefits of statins in people without established cardiovascular disease but with cardiovascular risk factors: meta-analysis of randomised controlled trials. $B M J$. 2009;338:b2376

13. Paciaroni M, Bogousslavsky J. Statins and stroke prevention. Expert Rev Cardiovasc Ther. 2009;7(10):1231-1243.

14. Ribeiro RA, Ziegelmann PK, Duncan BB, et al. Impact of statin dose on major cardiovascular events: a mixed treatment comparison metaanalysis involving more than 175,000 patients. Int J Cardiol. December 20, 2011. [Epub ahead of print.] 
15. Taylor F, Ward K, Moore TH, et al. Statins for the primary prevention of cardiovascular disease. Cochrane Database Syst Rev. 2011;1:CD004816.

16. Shafiq N, Malhotra S, Pandhi P, Grover A. The "Statinth" wonder of the world: a panacea for all illnesses or a bubble about to burst. J Negat Results Biomed. 2005;4:3.

17. Rahimi K, Majoni W, Merhi A, Emberson J. Effect of statins on ventricular tachyarrhythmia, cardiac arrest, and sudden cardiac death: a meta-analysis of published and unpublished evidence from randomized trials. Eur Heart J. February 3, 2012. [Epub ahead of print.]

18. Barter PJ, Caulfield M, Eriksson M, et al; ILLUMINATE Investigators. Effects of torcetrapib in patients at high risk for coronary events. $N E n g l$ J Med. 2007;357(21):2109-2122.

19. Kastelein JJP, Akdim F, Stroes ESG, et al; ENHANCE Investigators. Simvastatin with or without ezetimibe in familial hypercholesterolemia. N Engl J Med. 2008;358(18):1431-1443.

20. Taylor AJ, Villines TC, Stanek EJ, et al. Extended-release niacin or ezetimibe and carotid intima-media thickness. $N$ Engl J Med. 2009;361(22):2113-2122.

21. Ginsberg HN, Elam MB, Lovato LC, et al; ACCORD Study Group. Effects of combination lipid therapy in type 2 diabetes mellitus. $N$ Engl J Med. 2010;362(17):1563-1574.
22. Davignon J. Beneficial cardiovascular pleiotropic effects of statins. Circulation. 2004;109(23 Suppl 1):39-43.

23. Correa V Jr, Gus M, Fuchs FD. Does the blood pressure-lowering effect of statins contribute to their beneficial cardiovascular effects? Expert Rev Cardiovasc Ther. 2010;8(6):775-779.

24. Messerli FH, Pinto L, Tang SS, et al. Impact of systemic hypertension on the cardiovascular benefits of statin therapy - a meta-analysis. Am J Cardiol. 2008;101(3):319-325.

25. Thompson AM, Hu T, Eshelbrenner CL, Reynolds K, He J, Bazzano LA. Antihypertensive treatment and secondary prevention of cardiovascular disease events among persons without hypertension: a meta-analysis. JAMA. 2011;305(9):913-922.

26. Cannon CP, Shah S, Dansky HM, et al. Safety of anacetrapib in patients with or at high risk for coronary heart disease. $N$ Engl J Med. 2010;363(25):2406-2415.

27. Bays HE, Maccubbin D, Meehan AG, Kuznetsova O, Mitchel YB, Paolini JF. Blood pressure-lowering effects of extended-release niacin alone and extended-release niacin/laropiprant combination: a post hoc analysis of a 24-week, placebo-controlled trial in dyslipidemic patients. Clin Ther. 2009;31(1):115-122.
Vascular Health and Risk Management

\section{Publish your work in this journal}

Vascular Health and Risk Management is an international, peerreviewed journal of therapeutics and risk management, focusing on concise rapid reporting of clinical studies on the processes involved in the maintenance of vascular health; the monitoring, prevention and treatment of vascular disease and its sequelae; and the involvement of

\section{Dovepress}

metabolic disorders, particularly diabetes. This journal is indexed on PubMed Central and MedLine. The manuscript management system is completely online and includes a very quick and fair peer-review system, which is all easy to use. Visit http://www.dovepress.com/ testimonials.php to read real quotes from published authors. 